Alexandre E. Farias Frota

\title{
Avaliação de Opções Americanas Tradicionais e Complexas
}

\section{DISSERTAÇÃO DE MESTRADO}

\author{
DEPARTAMENTO DE ENGENHARIA INDUSTRIAL \\ Programa de Pós-Graduação em Engenharia de \\ Produção: Finanças e Análise de Investimentos
}




\section{Alexandre E. Farias Frota.}

\section{Avaliação de Opções Americanas Tradicionais e \\ Complexas}

\section{Dissertação de Mestrado}

Dissertação apresentada como requisito parcial para obtenção do título de Mestre pelo Programa de PósGraduação em Engenharia Industrial da PUC-Rio.

Orientador: José Paulo Teixeira Co-orientador: Tara Keshar Nanda Baidya 


\title{
Avaliação de Opções Americanas Tradicionais e Complexas
}

\begin{abstract}
Dissertação apresentada como requisito parcial para obtenção do título de Mestre pelo Programa de PósGraduação em Engenharia Industrial da PUC-Rio. Aprovada pela Comissão Examinadora abaixo assinada.
\end{abstract}

\author{
José Paulo Teixeira \\ Orientador \\ DEI-PUC.Rio \\ Tara Keshar Nanda Baidya \\ Co-orientador \\ DEI-PUC.Rio \\ Carlos Patrício Semanez \\ DEI-PUC.Rio \\ Marco Antônio Guimarães Dias \\ Petrobrás \\ Heber Moura \\ UECE/ Unifor \\ Prof. Ney Augusto Dumont
}

Coordenador (a) Setorial do Centro Técnico Científico - PUC-Rio

DEI-PUC.Rio, 27 de março de 2003 
Todos os direitos reservados. É proibida a reprodução total ou parcial do trabalho sem autorização da universidade, do autor e do orientador.

\section{Alexandre E. Farias Frota}

Graduou-se com honra ao mérito em Engenharia de Produção pela PUC.Rio em 2000. Recebeu certificado de excelência acadêmica nos anos de 1995 e 1996. Bolsista de intercâmbio universitário na Universidade de Oklahoma durante o ano de 1998. Participou dos projetos $\mathrm{PUC} / \mathrm{Petros}$ na área de asset management durante o ano de 2000 e PUC/Petrobrás sobre Opções Reais, durante o ano de 2002, coordenado pelo engenheiro Marco Antônio G. Dias. Publicou artigos na área de derivativos na Sociedade Brasileira de Finanças e Sociedade Brasileira de Pesquisa Operacional. Atualmente trabalha como analista de negócios na Diretoria de Manganês e Ligas da Companhia Vale do Rio Doce.

Ficha Catalográfica

Farias Frota, Alexandre

Aplicação de modelos flexíveis baseados em Simulação de Monte Carlo e Quase-Monte Carlo na avaliação de opções americanas tradicionais ou complexas.

[11] $143 \mathrm{f}$. il; $30 \mathrm{~cm}$

Dissertação (mestrado) - Pontifícia Universidade Católica do Rio de Janeiro, Departamento de Engenharia Industrial.

Incluí referências bibliográficas.

Opções Americanas; Complexas; Exóticas; Monte Carlo; Quase-Monte Carlo; Método dos Mínimos Quadrados; Métodos Numéricos, Processos Estocásticos. 


\section{Agradecimentos}

À minha esposa e melhor amiga Amabélia, por todo o amor, carinho e apoio. Sua presença foi imprescindível à realização deste trabalho.

Ao meu pai Manuel pela confiança depositada em mim e por estar sempre presente nos momentos que precisei.

À minha mãe Ruth, pelos momentos de tranqüilidade durante o ano de 2002.

Aos grandes amigos Viktor Nigri e Maurício Vidal cuja amizade e momentos de descontração enriqueceram esse mestrado.

Aos professores José Paulo Teixeira e Tara Nanda Badya pela inestimável colaboração.

À Marco Antonio Dias, Kátia Rocha e Edson cuja ajuda e incentivo foram de fundamental importância na elaboração deste trabalho.

Ao professor Carlos Patrício Samanez pelos ensinamentos que enriqueceram minha formação acadêmica.

Ao Conselho Nacional de Desenvolvimento Científico e Tecnológico (CNPq) pela ajuda financeira. 


\section{Resumo}

Farias Frota, Alexandre. Avaliação de Opções Americanas Tradicionais e Complexas. DEI-PUC.Rio, 2003. 143p. Dissertação de Mestrado Departamento de Engenharia Industrial, Pontifícia Universidade Católica do Rio de Janeiro.

A maioria das opções negociadas atualmente é do estilo americano, no entanto sua avaliação continua sendo uma tarefa bastante difícil, constituindo-se numa das áreas mais desafiadoras no campo de derivativos financeiros, particularmente quando existem vários fatores afetando o preço da opção. Isso ocorre basicamente porque os métodos de árvores binomiais e diferenças finitas tornam-se impraticáveis na avaliação de opções com mais de três fatores de incerteza. No presente trabalho, faz-se um estudo prévio dos modelos de precificação tradicionais, para posteriormente nos estendermos a modelos mais flexíveis desenvolvidos recentemente baseados em simulações de Monte Carlo e Quase-Monte Carlo, até então considerados inaplicáveis na avaliação de opções americanas. Nesse sentido, pretendemos comprovar a aplicabilidade e versatilidade dos modelos baseados em simulação na avaliação de opções americanas tradicionais ou complexas. Nossa análise baseia-se, sobretudo na ilustração de exemplos práticos, dando especial ênfase à implementação computacional e precisão dos modelos.

\section{Palavras-chave}

Opções Americanas; Complexas; Exóticas; Monte Carlo; Quase-Monte Carlo; Método dos Mínimos Quadrados; Diferenças Finitas, Métodos Numéricos; Processos estocásticos. 


\section{Abstract}

Farias Frota, Alexandre. Valuation of Ordinary and Complex American Options. DEI-PUC.Rio, 2003. 143p. MSc. Dissertation - Departamento de Engenharia Industrial, Pontifícia Universidade Católica do Rio de Janeiro.

The majority of the options negotiated nowadays are of the american style, however its valuation goes on being a very hard job, constituting themselves in one of the most challenging areas in the financial derivative field, particularly when there are several factors affecting the price of the option. It happens basically because the binominal trees and finite differences methods become impracticable in the valuation of options with more than three factors of uncertainty. In this work we are doing a previous study of the traditional methods of american option valuation for later extending this study to more flexible and newly developed models based on simulations of Monte Carlo and Quase-Monte Carlo, which up to the present have been considered inapplicable in the valuation of the american style options. In this sense we intend to prove the applicability and versatility of the models based on simulation in the valuation of traditional and complex american options. Our analysis is, above all based on the illustration of practical examples giving special emphasis to the computational implementation and accuracy of the methods.

\section{Keywords}

American Options; Complex; Monte Carlo; Least Square Method; Simulation; Finite Differences, Numerical Methods. 


\section{Sumário}

Lista de figuras, tabelas, ilustrações e quadros

Capítulo 1: Introdução

\section{CONCEITOS BÁSICOS}

Capítulo 2: Simulação de Monte Carlo 18

2.1. Conceitos Básicos 18

2.2. Esquemas Básicos de Precificação 21

2.2.1. Opção Européia $\quad 21$

2.2.2. Opção Barreira 23

2.2.3. Opção Asiática $\quad 24$

Capítulo 3: Métodos de Aceleração de Convergência 26

3.1. Técnicas de Redução de Variância 26

3.1.1.Variáveis Antitéticas 26

3.1.2. Variáveis de Controle 28

3.1.3. Estratificação 29

3.1.4. Importance Sampling 30

3.1.5. Latin Hipercube (LH) 32

3.2. Seqüências de Baixa Discrepância ou Quase-Monte Carlo (QMC) 34

3.2.1. Geração de Números Quase-Aleatórios Uniformes 36

3.2.2. Associação das Téc. de Redução de Variância e QMC 38

3.2.3. Homogeneidade das Seqüências em Altas Dimensões 39

3.2.4. QMC Híbrido 41

Capítulo 4: Opções Americanas 43

4.1 Conceitos Básicos 43

4.2. Formulação Matemática do Problema 44

4.3. Condição de Contorno Livre 46

4.4. Aproximações Analíticas 48 


\section{MODELOS TRADICIONAIS}

Capítulo 5: Modelo Binomial 49

5.1. Conceitos Básicos 49

5.2. Tipos de Modelos Binomiais $\quad 50$

5.2.1. Cox, Ross e Rubinstein (CRR) 50

5.2.2. Modelo de Jarrow e Rudd (JR) 52

5.2.3. Modelo de Hull e White (HW) 52

5.2.4. Modelo de Trigeorgis (TRG) 53

5.3. Variações do Modelo Binomial 53

5.3.1. Método dos Valores Médios (MVM) 53

5.3.2. Método Binomial Black-Scholes (BBS) 53

5.3.3. Método BBS com Extrapolação de Richardson (BBSR) 54

5.3.4. Método das Variáveis de Controle (MVC) 54

5.4. Resultados 55

Capítulo 6: Modelo de Diferenças Finitas 57

6.1. Escolha do GRID 58

6.2. Método Explícito 58

6.2.1. Instabilidade do Método Explícito 60

6.2.2. Interpretação Financeira da Instabilidade 60

6.3. Método Implícito 61

6.4. Método Crank-Nicholson 63

6.5. Gregas 65

6.6. Resultados $\quad 67$

MODELOS BASEADOS EM SIMULAÇÃO

Capítulo 7: Modelo de Grant, Vora e Weeks (GVW) 70

$\begin{array}{ll}\text { 7.1. Formulação do Problema } & 71\end{array}$

7.2. Esquema Gráfico de Precificação de uma Call Americana 72

7.3. Extensões do Modelo GVW 74

7.3.1. Técnicas de Quasi-Monte Carlo 74 
7.3.2. Método da Bisseção

7.3.3. Aplicar a Aproximação de Geske e Johnson (GJ) 75

7.4. Resultados

Capítulo 8: Modelo dos Mínimos Quadrados (LSM) 79

8.1. Formulação do Problema 80

8.2. Algoritmo LSM 83

8.3. Exemplo do Cálculo de uma Put Americana 85

8.4. Curva de Gatilho 90

8.5. Resultados 91

Capítulo 9: Avaliação de Opções Americanas Complexas 95

9.1. Modelo Jump-to-Ruin 95

9.2. Opções Barreira 97

9.3. Opções Asiáticas 100

9.4. Opções Lookback 102

9.5. Opções Dependentes de Múltiplos Ativos 104

9.6. Opções com Taxas de Juros Variáveis 106

9.6.1. Taxa de juros em função do tempo 107

9.6.2. Taxas de Juros estocásticas: Modelo CIR 109

9.7. Opções com Volatilidade Estocástica 111

Capítulo 10: Conclusão 113

$\begin{array}{ll}\text { Referências Bibliográficas } & 116\end{array}$

$\begin{array}{ll}\text { Apêndice A: Definições } & 120\end{array}$

A.1. Processo de Wiener 120

A.2. Lema de Itô 122

A.3. Dedução da Equação Diferencial de Black Scholes 123

A.4. Aproximação Analíticas 125

A.4.1. Barone-Adesi e Whaley 125

A.4.2. Bjerksund e Stensland 126

A.5. Aproximação de Geske e Johnson 127 
A.6. Equações de Diferenças: Série de Taylor

A.7. Métodos Iterativos

A.8. Método da Bisseção

A.9. Normalização de Seqüências $U \sim(0,1)$

134

A.10. Discrepância

135

A.11. Método dos Mínimos Quadrados

136

A.12. Polinômios

137

A.13. Fatoração LU

138

A.14. Números de Inicialização de Sobol

139

Apêndice B: Programas

140

B.1. Lista de Programas

141

B.2. Interface Computacional

142

B.2.1. Modelo de Diferenças Finitas

142

B.2.2. Modelo GVW

142

B.2.3. Modelo LSM

143 


\section{Lista de figuras, tabelas, ilustrações e quadros}

Figura 2.1- Esquema de precificação de uma opção européia.

Figura 2.2- Esquema de precificação de uma opção européia barreira. 24

Figura 2.3- Esquema de precificação de uma opção européia asiática. 25

Figura 3.1- Convergência do método Latin Hipercube. 33

Figura 3.2- Uniformidade das seqüências de números aleatórios. $\quad 34$

Figura 3.3- Distribuições geradas pelas seqüências de NA 35

Figura 3.4- Convergência dos métodos de MC e QMC. 35

Figura 3.5- Seqüências de baixa discrepância em altas dimensões. $\quad 39$

Figura 3.7- Uniformidade dos modelos de QMC e QMC-Híbrido. $\quad 42$

Figura 3.8- Simulação de preços no modelo de QMC e QMC-Híbrido. 42

Figura 4.1- Comparação entre opções americanas e européias. $\quad 44$

Figura 4.2- Soluções de Barone-Adesi e Bjerksund e Stensland. $\quad 48$

Figura 5.1- Convergência dos modelos binomiais CRR, JR, HW e TRG.55

Figura 5.2- Convergência dos modelos binomiais CRR, BBS, BBSR e MVM.

Figura 5.3- Precisão do modelo binomial CRR. 56

$\begin{array}{ll}\text { Figura 6.1- Convergência dos métodos de diferenças finitas. } & 67\end{array}$

Figura 6.2- Convergência do Modelo Crank-Nicholson. 67

Figura 6.3- Representação gráfica do GRID obtido no método de DF. 68

Figura 6.4- Demonstração da instabilidade do método. 68

Figura 6.5- Probabilidades negativas do método explícito. 69

Figura 6.6- Curvas de gatilho (condição de contorno livre). 69

Figura 7.1- Comparação entre opções bermudas e americanas. $\quad 70$

Figura 7.2- Simulação dos preços de uma ação com exercício antecipado. $\quad 76$

Figura 7.3- Curvas de gatilho para diversas datas de exercício antecipado. $\quad 77$

Figura 7.4- Curvas de gatilho com diferentes sementes de inicialização.77 
Figura 7.5- Precisão do modelo GVW.

Figura 8.1- Payoff's e curvas de continuação segundo o modelo LSM 92

Figura 8.2- Comparação das curvas de gatilho geradas pelos modelos de DF, LSM e GVW.

Figura 8.3- Curvas de gatilho para diversos intervalos de tempo. 93

Figura 8.4- Estratégia de exercício antecipado. 94

Figura 8.5- Informações sobre o risco. 94

Figura 9.1- Curvas de exercício médio do processo jump-to-ruin $\quad 97$

Figura 9.2- Curvas de exercício antecipado médio para uma opção americana put down-out.

Figura 9.3- Estratégia de exercício antecipado para as opções americanas barreira.

99

Figura 9.4- Curva de continuação de uma call americana asiática. 101

Figura 9.5- Estratégia de exercício antecipado para uma opção americana asiática. 102

Figura 9.6- Avaliação de uma opção americana lookback. 103

Figura 9.7- Estratégia de exercício para uma opção americana com múltiplos ativos. 106

Figura 9.8- Representação das curvas de juros USTS e DSTS. 108

Figura 9.9- Trajetórias de taxas de juros segundo o modelo CIR. 109

Figura 9.10- Taxas de desconto considerando juros estocásticos. 110

Figura 9.11- Simulação NGARCH. 112

Figura B.1- Interface computacional do programa American_DF. 142

Figura B.2- Interface computacional do programa American_GVW. 142

Figura B.3- Interface computacional do programa American_LSM. 143

Tabela 2.1- Convergência do modelo de SMC na precificação de uma put europeía.

Tabela 2.2- Convergência do modelo de SMC na precificação de uma put europeía barreira.

Tabela 2.3- Convergência do modelo de SMC na precificação de uma put europeía asiática. $\quad 28$

Tabela 3.2- Convergência da SMC com variáveis de controle. 29 
Tabela 3.3- Convergência da SMC com esratificação.

Tabela 3.4- Convergência da SMC com importance sampling. 32

Tabela 3.5- Convergência de QMC e variáveis de controle. 38

Tabela 7.1- Precisão do Modelo GVW.

Tabela 8.2- Modelo LSM em função do grau do polinômio linear utilizado na regressão.

Tabela 9.1- Opções americanas e européias cujos preços das ações seguem dois processos estocásticos diversos.

Tabela 9.2- Valores de opções americanas put up-out.

Tabela 9.3- Valores de opções americanas put up-out a medida que aumentamos o número de datas de exercício .

Tabela 9.4- Valores de opções americanas asiáticas . 101

Tabela 9.5- Valores de opções americanas lookback floating strike. 104

Tabela 9.6- Valores de opções americanas baseadas no valor mínimo dentre dois ativos.

Tabela 9.7- Valores de opções americanas baseadas no valor máximo dentre três ativos correlacionados.

Tabela 9.8- Valor de opções americanas com juros variáveis 108

Tabela 9.9- Opções americanas com juros estocásticos. 110

Tabela 9.10- Opções americanas c/ volatilidade estocástica. 112

Tabela A.1- Parâmetros do método de inversão de Moro. 135

Tabela A.2- Números de inicialização da seqüência de Sobol com seus respectivos polinômios primitivos até a dimensão 32 .

llustração 3.1- Esquema do método de estratificação. 30

llustração 3.2- Esquema do método Latin Hipercube. 33

llustração 3.3- Esquema do modelo de QMC-Híbrido. 41

llustração 4.1- Esquema gráfico da condição de contorno livre. 47

llustração 5.1- Esquema gráfico de uma árvore binomial. 49

llustração 6.1- Demonstração do GRID e dos métodos de DF 58

llustração 7.1- Esquema GVW: PASSO 1

llustração 7.2- Esquema PASSO 2

llustração 7.3- Esquema PASSO 3 
llustração 7.4- Esquema PASSO $4 \quad 74$

llustração 7.5- Esquema do método da bisseção. 75

Quadro 8.2- Exemplo modelo LSM: Valores de exercício. 86

Quadro 8.3- Exemplo modelo LSM: Regressão no instante $t_{2}$. 87

Quadro 8.4- Exemplo modelo LSM: Estratégia de exercício em $t_{2} . \quad 87$

Quadro 8.5- Exemplo modelo LSM: Regressão no instante $t_{1}$. 88

Quadro 8.6- Exemplo modelo LSM: Estratégia de exercício em $t_{1}$. $\quad 89$

Quadro B.1- Lista de programas desenvolvidos. 141 